\title{
Aplicación de sistemas programables para los sistemas computacionales y robóticos animatronics
}

Jeison Andrés Orjuela Parra*

\section{Resumen}

En este artículo se presentan los resultados obtenidos durante el desarrollo de un sistema que, al reunir diversos componentes tecnológicos, es capaz de generar movimientos articulares similares a los realizados por el cuerpo humano. A su vez, el documento versará sobre la integración de protocolos de comunicación cuya función es controlar remotamente dicho sistema y, aún más, sobre el proceso de incorporación de diferentes unidades electrónicas que dotarían al sistema de robustez tal que lo harían análogo a uno industrial. También tocará el asunto de la implementación de metodologías capaces de adaptarse al diseño estructural mediante componentes gráficos, análisis de comportamientos y simulación mediante aplicaciones virtuales o matemáticas. Dispuesto así, a lo largo de este texto se intentará evidenciar la importancia de la sinergia de diferentes disciplinas del conocimiento, su utilidad, su evaluación y su necesidad comercial en la construcción de animatronics, a saber, robots capaces de simular movimientos de la naturaleza que se están incorporando paulatinamente en el mundo contemporáneo.

Palabras clave: amplificación, control análogo, fpga, programación, robótica, sistema Scada

\begin{abstract}
This article presents the results obtained during the development of a system that, by bringing together various technological components, is capable of generating joint movements similar to those carried out by the human body. In turn, the document will deal with the integration of communication protocols whose function is to remotely control that system and, even more so, on the process of incorporating different electronic units that would endow the system with robustness such that they would make it analogous to an industrial one. It will also address the issue of the implementation of methodologies capable of adapting to structural design through graphic components, behavioral analysis and simulation through virtual or mathematical applications. Throughout this text, I will try to show the importance of the synergy of different disciplines of knowledge, their usefulness, their evaluation and their commercial need in the construction of animatronics, robots capable of simulating movements of nature that are gradually being incorporated into the contemporary world.
\end{abstract}

Keywords: Amplification, Analog Control, fpga, Programming, Robotic, Scada System

* Estudiante de Ingeniería Electrónica de la Corporación Unificada Nacional de Educación Superior (CUN). Contacto: jeison. orjuela@cun.edu.co 


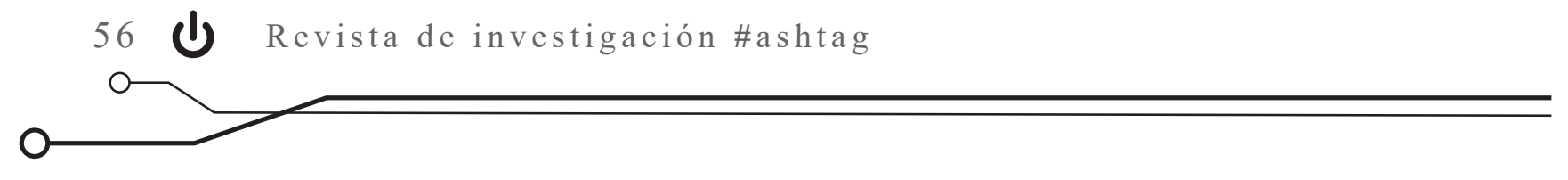

\section{Introducción}

Con el objetivo de establecer la sinergia de diferentes componentes electrónicos y su aplicabilidad en el sector industrial, se realiza la adaptación e incorporación de diversas disciplinas y conocimientos proporcionados por la ciencia computacional, la electricidad y especialmente la electrónica, en la construcción de un robot animado denominado animatronic (término usado por las industrias de automatización, producción visual y de efectos especiales). Este será el objeto de estudio de la presente disertación. En esencia, la finalidad es evaluar y analizar los diferentes sistemas que lo integran. Dispuesto así, este proyecto, de un lado, denota el seguimiento a la continuidad de competencias de cada asignatura cursada por separado en la academia, y, de otro, busca adaptarlas y ponerlas en diálogo entre sí en función de un macroproyecto.

Para ello, se mostrará el paso a paso que requiere la elaboración de una máquina animada: diseño de modelamiento gráfico y mecánico; estudio de su comportamiento físico y electrónico; aplicación de los conceptos de programación; uso de sistemas programables (como tarjetas FPGA, microcontroladores, tarjetas comerciales Arduino, PSoC, entre otros elementos); su sincronización para el control de mecanismos, y ajuste de la conectividad de los diversos protocolos de comunicación convencional. Esto permitirá comprender los comportamientos esperados del sistema robótico a la luz de las necesidades planteadas y especialmente ante los retos que conllevó la inclusión de cada dispositivo, su ensamble y, aún más, garantizar su correcto funcionamiento.

Respecto al animatronic presentado en este artículo, es importante mencionar que no solo pretende simular el comportamiento humano, sino también el entorno que lo rodea. Un ejemplo claro que ilustra los logros alcanzados por la robótica son los parques temáticos a nivel mundial, pero, en especial, los de Orlando (Florida), entre ellos, Magic Kingdom, Epcot, Busch Gardens, SeaWord, etc. Ahora, dos atracciones muy significativas por su extendido uso de animatronics que simulan movimientos humanos son A Small World, en Magic Kingdom, y Jurassic Park, en Universal Studios.

Amparado en la guía de autores representativos en la materia como Ollero (2001) o Balcells (1999), en este proyecto se implementaron técnicas de control de diferentes elementos mecánicos a través de la electrónica y la programación, pero aún se deben enfrentar problemas como la falta de un control comercial del sistema de potencia, pues el sistema actualmente implementado no cuenta con un control automático del consumo energético. Con respecto a los sensores y actuadores que regulan el comportamiento del animatronic, aún presenta falencias en su estructura física, faltan elementos mecánicos que compongan un rostro con rasgos más humanos, y no cuenta con un sistema de inteligencia artificial por lo que todos sus movimientos son controlados por un operador. 


\section{Formulación del problema}

El presente trabajo pretende responder y aportar información a la comunidad académica en relación a la siguiente pregunta: ¿cómo realizar un mecanismo robótico que use sistemas de lógica computacional para el control de dispositivos electro-mecánicos que simulen el cuerpo humano? La pregunta de investigación planteada invita a relacionar los elementos programables disponibles en el mercado convencional, en particular, aquellos que maximizan la funcionalidad y coadyuvan en un sin fin de utilidades. Por

\section{Marco teórico}

\section{Historia de la animatrónica}

El primer registro que se tiene en la historia de un animatronic aparece en 1515: el león autómata de Leonardo Da Vinci, construido para facilitar las conversaciones de paz entre el rey de Francia y el papa León X. El animal, mediante diversos mecanismos, anduvo de una habitación a otra, hasta donde se encontraba el monarca. Abrió su pecho y todos pudieron comprobar que estaba lleno de lirios y otras flores que representaban un antiguo símbolo de Florencia (el león) y la flor de lis que Luis XII regaló a la ciudad como señal de amistad.

Se dice que la animatrónica fue desarrollada por Walt Disney en los sesenta. El término abreviado fue acunado originalmente de los audio-animatronics de Walt Disney, que luego fue usado para describir a los personajes mecanizados. Algunos ejemplos de los animatrónicos de Disney se encuentran en The Hall of Presidents y en Disneyland. En la feria mundial realizada en Nueva York en 1964, la Disney Company presentó una marioneta del presidente Lincoln cuyo cuerpo, eso, es importante conocer la implementación de cada uno, así como evaluar su desempeño y alcances. Por otra parte, el presente trabajo evidenciará la posibilidad de articular diferentes dispositivos electrónicos en un sistema que permita el desarrollo de una maquina compuesta por diversos elementos mecánicos entrelazados. De esta manera, se mostrará la semejanza con el cuerpo humano y su aplicación a nivel industrial.

lenguaje y el movimiento facial cazaban perfectamente con el audio (Hamilton, 1998).

Un hombre clave en la historia de la animatrónica fue el estadounidense Stan Winston, quien empezó su carrera como maquillador para la Disney Company en el añode 1969. Allí aprendió el oficio de la animatrónica. En 1970 se independizó y formó su propia compañía: Stan Winston Studios. Desde entonces se convirtió en protagonista, tras bambalinas, de los efectos especiales más famosos en la historia cinematográfica; es el responsable de los efectos y animatrónicos de la saga Terminator (I, II y III) Alien (I y II), Depredador (I y II), Jurassic Park (I, II y un poco en la parte III) y dio vida al oso Teddy en la película Inteligencia artificial. A pesar de su fallecimiento en el año 2008, su equipo ha continuado su legado $\mathrm{y}$, de la mano de los avances en técnicas de control y nuevos materiales, ha mantenido su lugar privilegiado con sus animatrónicos en el mundo del espectáculo. 


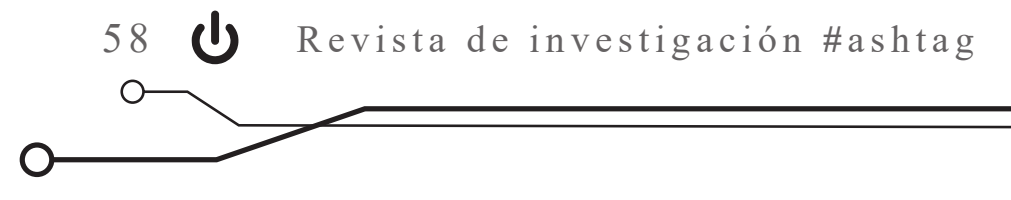

Desde 1977 hasta la actualidad, la empresa californiana Garner Holt Productions INC18 ha fabricado más de 3000 figuras animatrónicas para parques temáticos y museos alrededor del mundo. Sus figuras animatrónicas toman la forma de personajes de caricaturas, personas y animales. Actualmente, su proceso de producción comienza con el diseño tridimensional en Solid Works: escultores especializados con imágenes detalladas se encargan de que el animatrónico sealo más parecido al referente real; logran su funcionamiento mediante sistemas neumáticos, hidráulicos y electrónicos que actúan como

\section{Aplicación de la animatrónica}

La principal utilidad de la animatrónica se evidencia en el campo de la cinematografía y los efectos especiales, aunque también se emplea con frecuencia en los parques temáticos y en otras ramas de la industria del entretenimiento. Su principal ventaja respecto a las imágenes generadas por ordenador o la técnica de stop motion es que no consiste en una simulación de la realidad, sino que presenta ante la cámara objetos auténticos que se mueven en tiempo real. Con los años, la tecnología que da soporte a la animatrónica se ha ido haciendo más y más compleja, hecho que ha posibilitado la construcción de marionetas cada vezmás realistas y aparentemente vivas.

Los muñecos animatrónicos se emplean en cine y televisión para representar personajes que no existen en el mundo real o que están en situaciones de riesgo, así como escenas en las que no

\section{Elementos electrónicos. La tarjeta PSOC}

El PSoC (programmable system-on-chip) es un sistema que ofrece novedosas capacidades integradas en un solo chip. Estos son fabricados por la músculos y esqueletos de acero y aluminio; por su parte, los controles computarizados manejan los perfiles de movimiento y sonido.

Hoy por hoy, la animatrónica se ha desarrollado como una carrera que requiere la combinación de talentos en ingeniería mecánica, eléctrica y electrónica; escultura; tecnologías de control; radio control y aerografía. De la mano de la aparición de los efectos digitales, los animatrónicos han hecho parte de la historia cinematográfica.

sería aconsejable el uso de actores o animales, o en las que las acciones a realizar no podrían conseguirse con personas o animales auténticos. La animatrónica de hoy en día emplea dispositivos controlados por ordenador, así como controles por radio o manuales. Los movimientos específicos se consiguen mediante motores eléctricos, cilindros neumáticos o hidráulicos y mecanismos controlados por cable. El tipo de elementos a utilizar se decide en función de los parámetros del personaje, los movimientos concretos que se requieren y las limitaciones del proyecto.

A mediados de los ochenta, se planteó que el uso de robots podría incluirse en las tareas en las que el ser humano asume importantes riesgos o en las que sus capacidades se ven limitadas por factores como la fuerza o la precisión. A estos se les denominó robots de servicio.

compañía norteamericana Cypress Semiconductor, con un moderno método de adquisición, procesamiento y control de señales que los dota 
de una excelente precisión. Incluyen, además, un arreglo de bloques digitales (UDB) y analógicos que hacen del PSoC una muy buena propuesta para el desarrollo de proyectos de ingeniería, pues le proporcionan al sistema la capacidad de establecer cualquier función, en cualquier terminal del circuito integrado. Son, en resumen, muy versátiles.

Los PSoC emplean una arquitectura configurable para el control del diseño embebido, equivalente a un FP-ASIC (field programable application-specific

\section{Servomotor SG90}

El servomotor SG90 es un servo miniatura de gran calidad y diminutas dimensiones, además es bastante económico. Funciona con la mayoría de tarjetas electrónicas de control con microcontroladores y además con la mayoría de los sistemas de radio control comerciales. Este tipo de servo es ideal para las primeras experiencias de aprendizaje y prácticas con servos, ya que sus requerimientos de energía son bastante bajos y se permite alimentarlo con la misma fuente de alimentación que el circuito de control. (Imperial College, 2016)

\section{Solución propuesta}

En primer lugar, se analizó el comportamiento humano con el fin de realizar un diseño acorde al sistema fisiológico del ser humano. Por lo tanto, con base en trabajos como el de Correa (2007), se realizaron los siguientes pasos para su implementación.

Con el objetivo de realizarun sistema robótico, se planteó desarrollar un sistema capaz de simular integrated circuit) pero con la ventaja del tiempo de implementación. Los dispositivos PSoC integran circuitos digitales y analógicos configurables, controlados por un microcontrolador interno; de modo que proveen tanto una capacidad mejorada para la revisión de los diseños, como la disminución del número de componentes usados. Un solo PSoC puede integrar hasta cien funciones periféricas y ahorrar de esta manera tiempo de diseño, espacio físico y consumo de energía, mientras que mejora la calidad del sistema y reduce su costo (Cypress, 2016).

El objetivo de usar el servo es controlar la posición de la carga mecánica de acuerdo con la posición de referencia mediante un par de potenciómetros que funcionan como un dispositivo de medición de error y convierten las posiciones de entrada $\theta r(t)$ y salida $\theta c(t)$ en señales eléctricas proporcionales.

La posición angular $\theta \mathrm{c}(\mathrm{t})$ del potenciómetro de salida genera un potencial eléctrico $\mathrm{Ec}(\mathrm{t})$ que luego alimenta el terminal negativo de un amplificador diferencial dentro del servo para así realizar la comparación con la posición de entrada y cerrar el bucle de control de posición.

el comportamiento humano mediante un rostro humanoide. Este, además, incluiría una parte del sistema cardiaco y torácico. Por otra parte, se elaboró una mano robótica para darle más realismo al animatronic. La figura 1 muestra el modelo gráfico empleado para la impresión 3D con ácido poliláctico (PLA, por su sigla en inglés), de los elementos involucrados en el rostro del animatronic. 


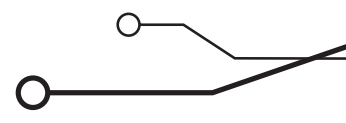

Figura 1. Modelo 3D del rostro

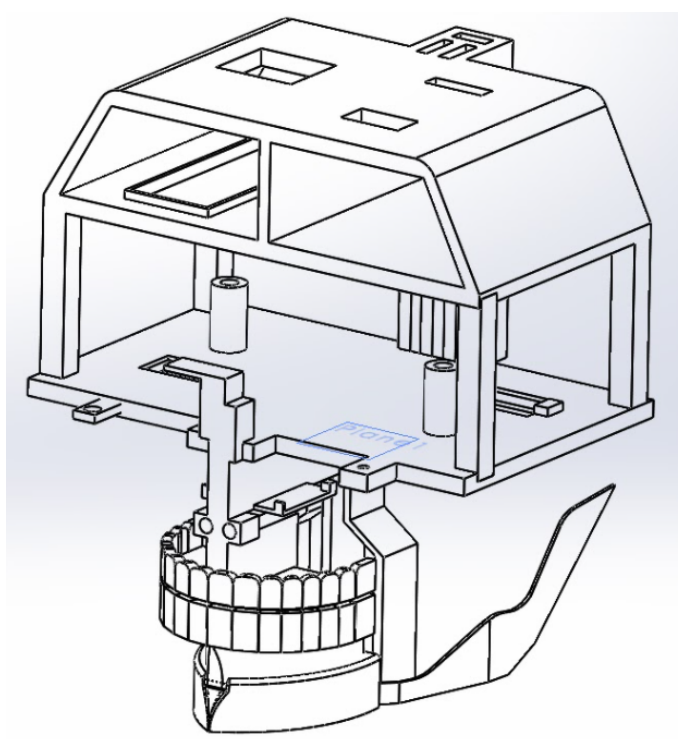

Fuente: elaboración propia

Se realizó el modelamiento pensando en las siguientes articulaciones:

1. Ojos: movimiento horizontal y vertical manipulado por protocolo bluetooth. Utiliza 2 servomotores SG90 y su control depende de una tarjeta Arduino, así como de una aplicación móvil diseñada por APP Inventor.

2 Pómulos: los pómulos están controlados por 2 servomotores conectados a la tarjeta FPGA, la cual recibe un pulso microeléctrico adaptado mediante instrumentación industrial para percibir los movimientos del antebrazo humano.

3. Maxilar inferior: el maxilar interior es controlado por un servomotor conectado a un sistema de amplificación y modulado por un micrófono y un circuito 555, el cual controla su apertura mediante PWM, en concordancia con los decibeles captados en el ambiente. Simula el movimiento y gesticulación del habla.
4. Cuello: el cuello está conectado a dos servomotores conectados al controlador Arduino, el cual se comunica vía bluetooth mediante una aplicación móvil. Realiza movimientos tanto horizontales como verticales.

5. Párpado: el sistema del párpado está conectado como en los anteriores casos a un servo motor SG90, pero, a diferencia de los demás, su control es independiente y está configurado mediante la tarjeta FPGA, la cual establece dos pulsos en el tiempo (uno con una frecuencia de $20 \mathrm{~Hz}$ y otra con una frecuencia de $30 \mathrm{~Hz}$ ). Cuando se encuentran en el tiempo correcto, generan un pulso o nivel alto relacionado con una señal discreta. Su composición está basada en el uso de las compuertas lógicas (Bouchet, 1979).

La figura 2 muestra los resultados de la implementación de la cabeza del animatronic.

Figura 2. Impresión real

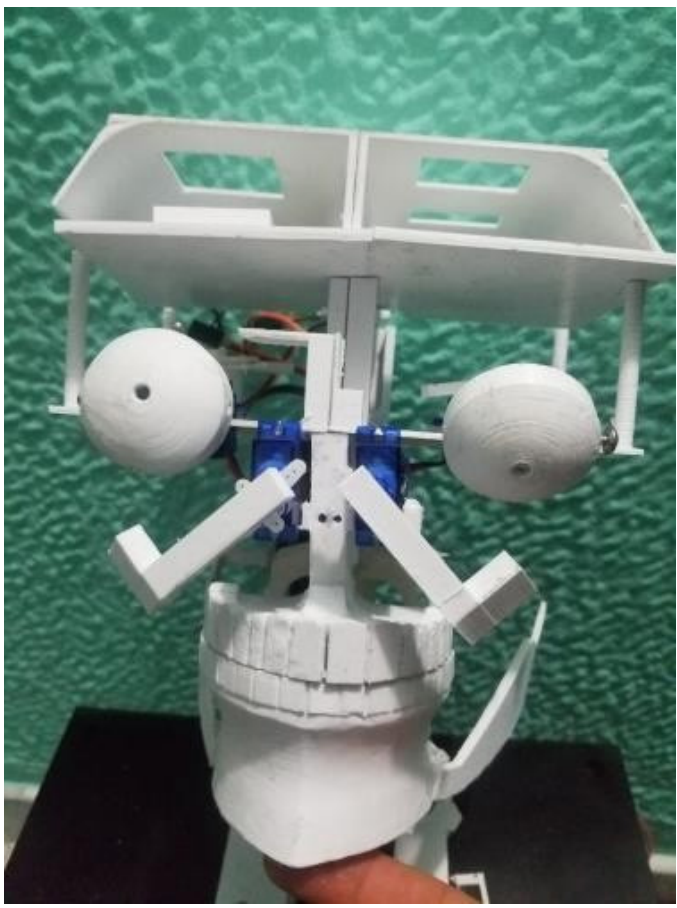

Fuente: elaboración propia 
6. Corazón: se encuentra visualmente representado en gran tamaño. Es realmente llamativo y simula los procesos cardíacos.

Figura 3. Sistema tórax

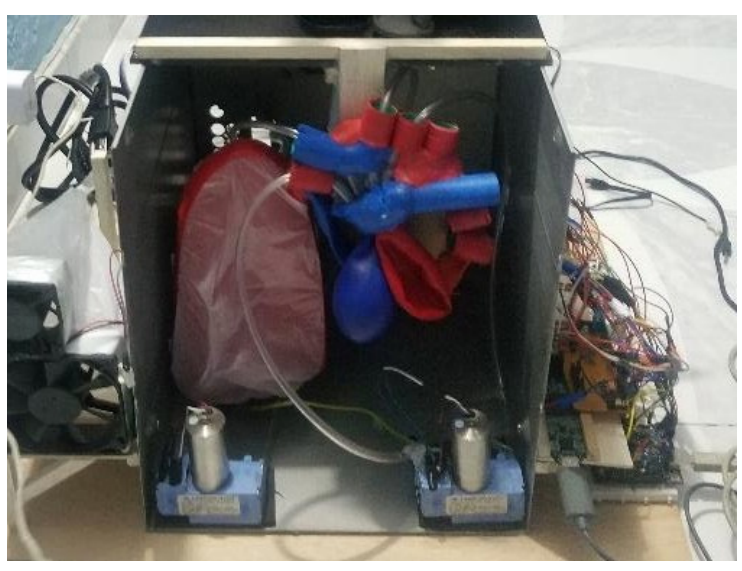

Fuente: elaboración propia

El corazón está controlado mediante dos ciclos en el tiempo a frecuencias diferentes y permite ser controlado, así mismo como la arritmia, mediante un módulo RF. Esto posibilita recibir diferentes pulsos decodificados en la tarjeta FPGA. Este pulso procesado permite generar una salida digital que es enviada a un módulo puente $\mathrm{H}$ conectado a dos electroválvulas que simulan el pulso cardíaco. Un sistema similar se encuentra en los ventiladores de $12 \mathrm{v}$ conectados con el pulmón, lo que simula la inhalación y exhalación. La figura 3 muestra la implementación del tórax.

El control se realiza mediante Labview ${ }^{\circledR}$. Este software visualiza un ambiente gráfico en donde se evalúa el comportamiento del sistema conectado, y además de eso genera alarmas dependiendo como esté funcionando.
Figura 4. Interfaz Labview

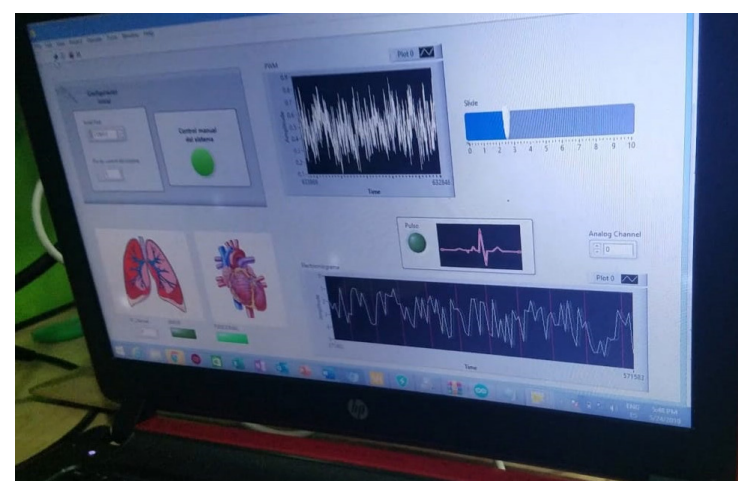

Fuente: elaboración propia

En la figura 4 puede verse el ambiente gráfico disponible para el sistema. Allí se tiene un control mediante PWM del destello de los ojos que controla su luminosidad e incluso el efecto robótico de destello de luz. También se tiene una alarma que evidencia, de color verde, el control cardíaco y el sistema pulmonar. Por otra parte, se tiene una adaptación de la señal mioeléctrica, tomada por el amplificador de instrumentación implementado para la adquisición de señales de pulsos eléctricos corporales, así como la activación o desactivación de emergencia del dispositivo cardiaco y pulmonar, por si presenta alguna falla.

7. Control de seguridad: el sistema de control de seguridad es un teclado matricial de $4 \times 4$ conectado a la tarjeta FPGA. Este sistema se encuentra decodificado por esta, lo que permite recibir de manera correcta los números digitados con la salida lógica en el sistema. Por otra parte, el sistema de almacenamiento mediante flip flop tipo $\mathrm{D}$ almacena una clave mediante programación y permite compararla con la digitada en tiempo real; si la clave es correcta permitirá la activación del sistema, pero, si es equivocada, la tarjeta FPGA informará que es incorrecta mediante el encendido de un testigo de color rojo. 


\section{$62 \bigcup$ Revista de investigación \#ashtag}

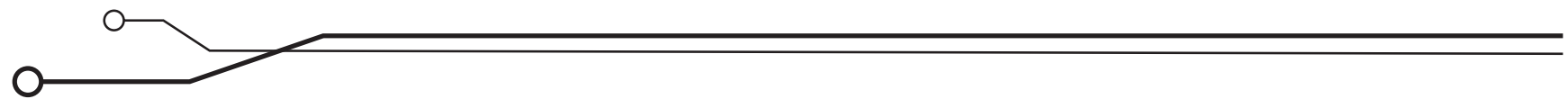

8. Mano robótica: la mano robótica tiene diez servomotores conectados a una tarjeta FPGA, la cual recibe la señal de un microcontrolador que, a su vez, es controlado por un sistema Scada. Este último permite controlar los movimientos de la mano mediante el puerto UsB, mientras que el sistema computacional brinda la posibilidad de controlar los diferentes movimientos posibles (Pérez y Mendoza, 2008). La figura 5 muestra la implementación de la mano pensando en los movimientos de la figura 6.

Figura 5. Mano robótica

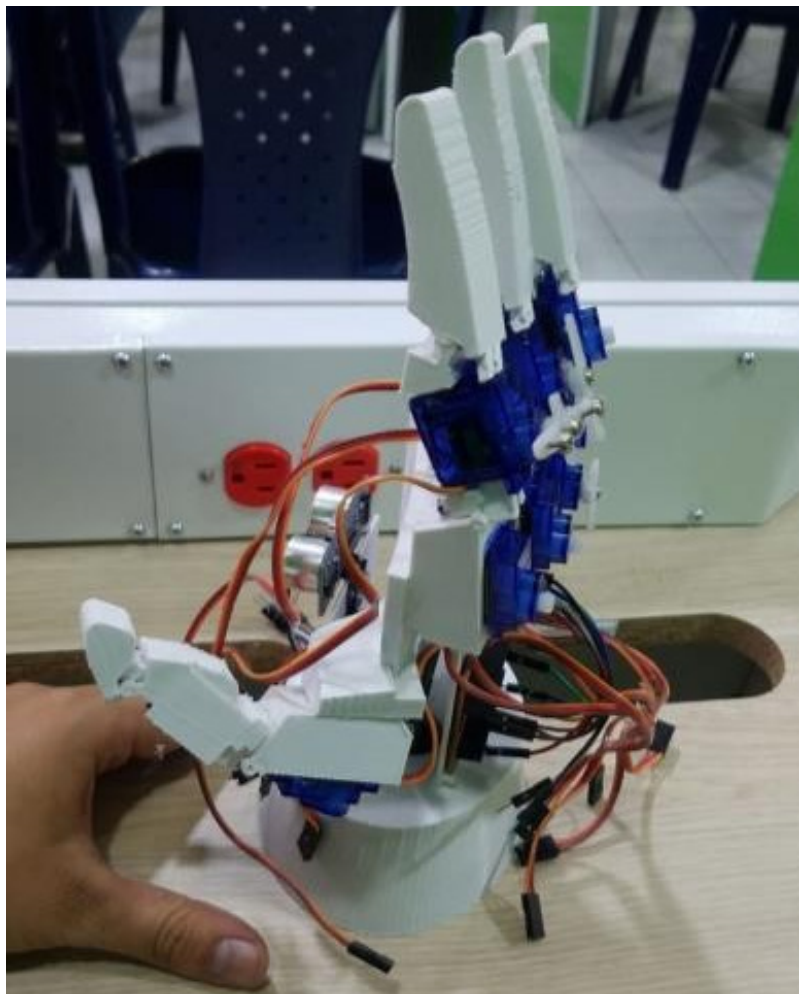

Fuente: elaboración propia

Figura 6. Posibles movimientos de la mano

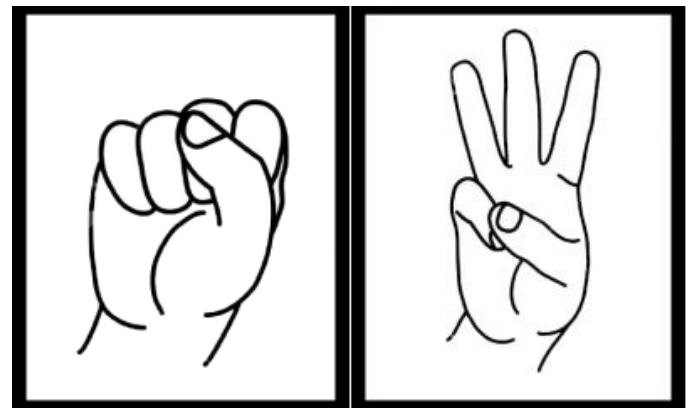

Fuente: elaboración propia 


\section{Pruebas}

Una vez construida la estructura física del animatronic, se implementó el mecanismo basado en servomotores para controlar la articulación de los ojos (figura 7).

Figura 7. Sistema de control de los ojos

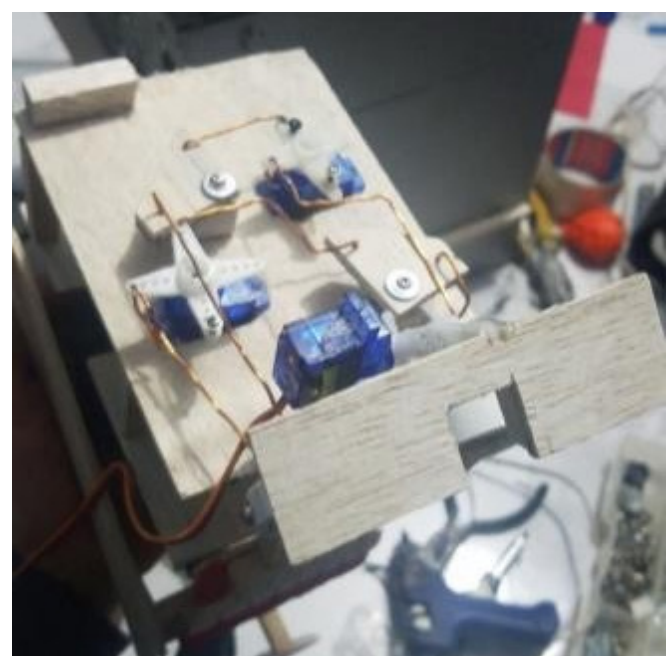

Fuente: elaboración propia

De igual manera, se desarrolló un sistema de control del sistema del puente $\mathrm{H}$ conectado a las electro válvulas y los motores DC $12 \mathrm{~V}$ para el control del tórax, y otro para el control de la mandíbula y la salida de audio (figuras 8 y 9).

Figura 8. Control puente $\mathrm{H}$

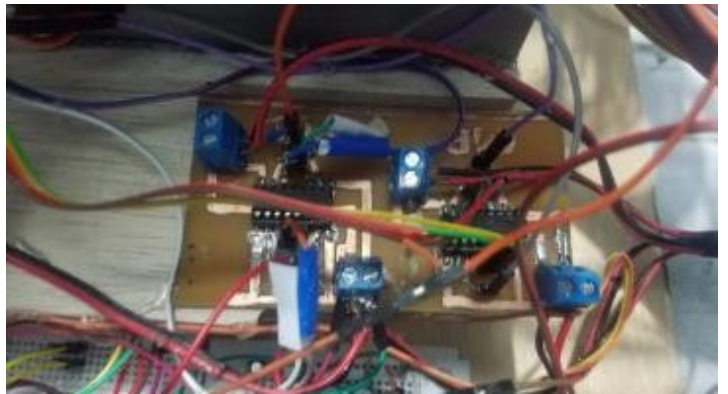

Fuente: elaboración propia
Figura 9. Sistema de amplificación para el maxilar inferior

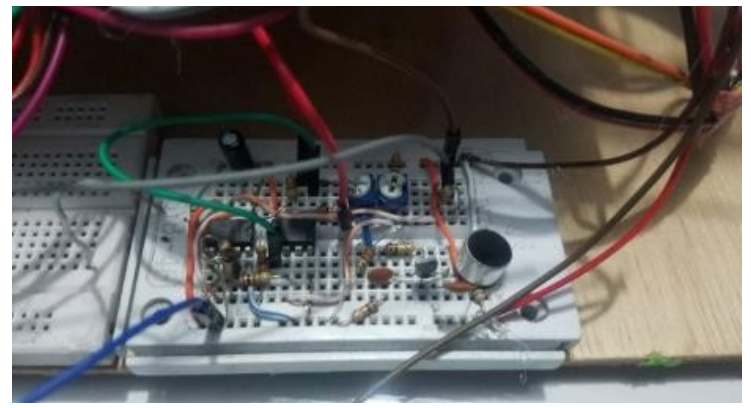

Fuente: elaboración propia

Esto completa el sistema que permite el habla del dispositivo (conectado a un parlante). Este se encuentra asociado a un módulo ISD que permite grabar un sonido y, cada vez que recibe un pulso, lo reproduce para aparentar realismo. Por último, se desarrolló el sistema de control remoto basado en tecnología de radio frecuencia y módulo bluetooth (figura 10).

Figura 10. Sistema de recepción modulo RF y bluetooth

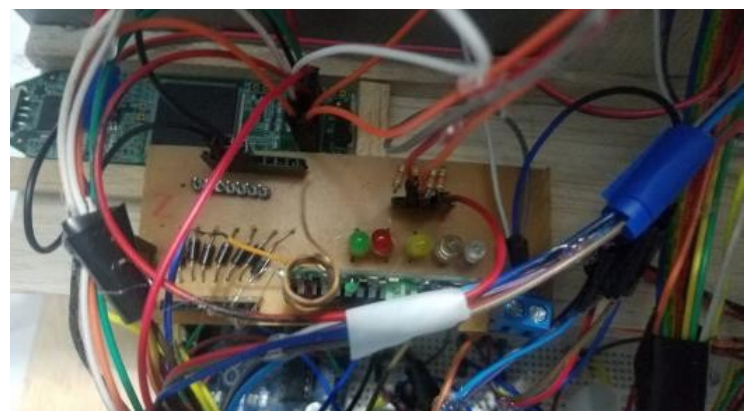

Fuente: elaboración propia

En la fase de pruebas, se evidenció una independencia del robot de hasta 2 metros sin ningún tipo de interferencia en la emisión de las señales por control remoto. Finalmente se integraron todos los sistemas al módulo FPGA (figura 11). 


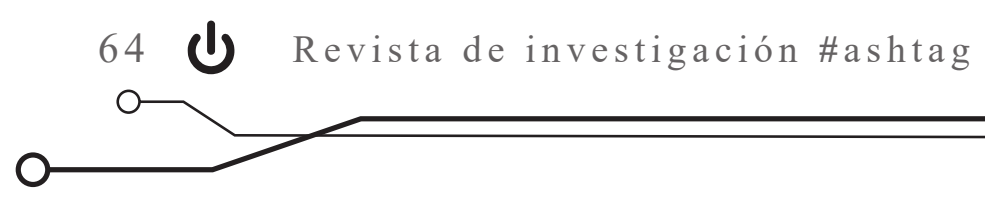

Figura 11. Implementación de tarjeta FPGA

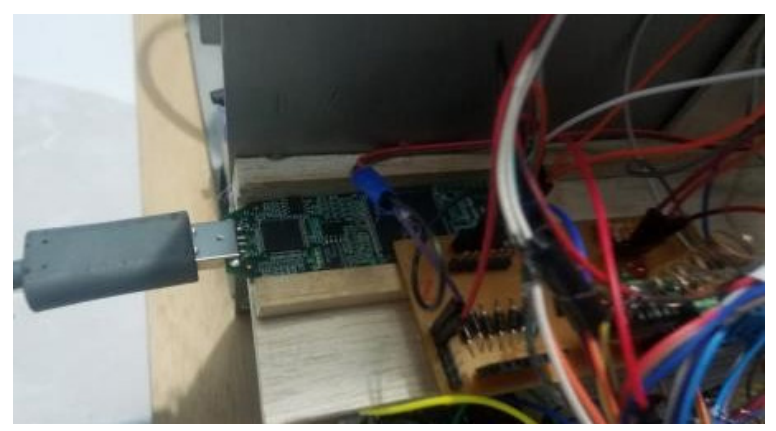

Fuente: elaboración propia

Esta tarjeta mostró gran eficiencia en la articulación de los circuitos programados, así como sinergia con otros elementos, y practicidad para la emisión o recepción de señales.

\section{Conclusiones}

Se cumplió el objetivo de realizar un sistema capaz de interactuar con diferentes sistemas lógicos computacionales, para generar diferentes formas de locomoción y con la posibilidad de que sus diferentes mecanismos sean manipulados remotamente.

Se obtuvo respuesta satisfactoria de la sincronización de la tarjeta FPGA y el microcontrolador; un correcto funcionamiento del sistema diseñado en Labview para el monitoreo de sensores y sistemas, y la aplicabilidad de sistema Scada de manera remota, lo que evidenció resultados eficientes. Por otra parte, se obtuvo el correcto
Gracias a las pruebas realizadas podemos determinar que el sistema animatrónico logra cumplir el objetivo, debido a que controla los diferentes mecanismos disponibles en el sistema: mano, pómulos, corazón, ojos, cuello, párpados. Así mismo, se sincroniza mediante la tarjeta Arduino UNO con el bluetooth y el módulo RF. Este último recibe los datos emitidos por medio remoto (como aplicación móvil o control RF) y los envía a la tarjeta FPGA para su correcto control. Por otra parte, se sincroniza con los ordenadores a través de un sistema de monitoreo, tipo Scada, capaz de controlarlo enviando señales, analizándolas y controlándolas, incluso remotamente. Para finalizar, el sistema del habla es controlado por los decibeles del ambiente y con las señales mioeléctricas para el control de parpados y otras partes del robot.

ángulo de apertura y cierre de la mano robótica tras ajustarlo con la intención de imitar el movimiento humano. Se demuestra que los protocolos de comunicación como el RF y bluetooth reaccionaron eficientemente, lo que posibilitó tener control inalámbrico del sistema.

Finalmente, se evidenció la aplicabilidad de la tarjeta FPGA para desarrollar el sistema deseado mediante su programación. Se reconoce que la tarjeta de libre desarrollo es de gran utilidad y ofrece ventajas considerables, en contraste con las convencionales que requieren licenciamiento. 


\section{Referencias}

Balcells. J. (1999). Autómatas programables. Barcelona: Alfaomega.

Bouchet, C. (1979) Anatomía de la cara, cabezayórganos delos sentidos. Madrid:Editorial Panamericana,

Correa, D. (2007). Diseño y construcción de mano animatrónica [trabajo de grado].Universidad EAFIT, Medellín, Colombia.

Cypress Developer Community. (2016). Changing the Target Device (PSoC ${ }^{\circledR} 3 /$ PSoC 5LP) in PSoC Creator. Recuperado de https:// bit.ly/35cm0kH

Hamilton, J. (1998). Efectos especiales en el cine y la televisión. Londres: Dorlyng Kindersley Limited.

Imperial College of London, Department of Electrical and Electronic Engineering. (2016). Servo Motor SG90 Data Sheet. Recuperado de https:/ / bit.ly/3nIkL2Z

Ollero, A. (2001). Robótica, manipuladores y robots móviles. Barcelona: Alfaomega.

Pérez, I. y Mendoza, H. (2008). Desarrollo de una mano robótica con capacidad de manipulación. Telematique. Revista Electrónica de Estudios Telemáticos, 7(1), 180-207. Recuperado de https:/ / bit.ly/2F07jGh 